\title{
The GMD Method for Inductance Calculation Applied to Conductors with Skin Effect
}

\author{
H. A. Aebischer ${ }^{1 *}$, B. Aebischer ${ }^{2}$ \\ ${ }^{1}$ LEGIC Identsystems AG, Switzerland \\ ${ }^{2}$ Hexagon Technology Center GmbH, Switzerland \\ *corresponding author, E-mail: hubert.aebischer@legic.com
}

\begin{abstract}
The GMD method (geometric mean distance) to calculate inductance offers undoubted advantages over other methods. But so far it seemed to be limited to the case where the current is uniformly distributed over the cross section of the conductor, i.e. to DC (direct current). In this paper, the definition of the GMD is extended to include cases of nonuniform distribution observed at higher frequencies as the result of skin effect. An exact relation between the GMD and the internal inductance per unit length for infinitely long conductors of circularly symmetric cross section is derived. It enables much simpler derivations of Maxwell's analytical expressions for the GMD of circular and annular disks than were known before. Its salient application, however, is the derivation of exact expressions for the GMD of infinitely long round wires and tubular conductors with skin effect. These expressions are then used to verify the consistency of the extended definition of the GMD. Further, approximate formulae for the GMD of round wires with skin effect based on elementary functions are discussed. Total inductances calculated with the help of the derived formulae for the GMD with and without skin effect are compared to measurement results from the literature.

For conductors of square cross section, an analytical approximation for the GMD with skin effect based on elementary functions is presented. It is shown that it allows to calculate the total inductance of such conductors for frequencies from $\mathrm{DC}$ up to $25 \mathrm{GHz}$ to a precision of better than $1 \%$.
\end{abstract}

\section{Introduction}

There are basically three methods to calculate inductance: Firstly, one can use the definition of the magnetic energy and integrate the square of the magnetic induction over the whole space. Secondly, one can integrate the magnetic induction over the surface spanned by the loop circuit to be analyzed and divide the resulting magnetic flux by the total current. In both methods the internal inductance, i.e. the contribution from the magnetic induction inside the conductor, must be calculated separately. The calculation of the inductance of loop circuits by these two methods requires the use of numerical techniques like finite elements. The computational effort is huge. Thirdly, there is the method based on the magnetic vector potential. With its help, the magnetic energy can be written as a volume integral over the product of the magnetic vector potential and the current density. Since current flows only within the conductor, the integral can be reduced from the whole space to the volume of the conductor. Substituting the vector potential by the solution of its Poisson equation leads to the definition of inductance as a double volume integral over the conductor. For the case of straight conductors with uniform distribution of current, this leads to the GMD method [1] - [6], which was invented by Maxwell ([1], §692). Its main parameter is the GMD (geometric mean distance) of the conductor cross section to itself.

The GMD method offers substantial advantages over the other methods: It provides general analytical expressions for the total (i.e. external plus internal) partial inductance of a single straight conductor, valid for any cross-sectional shape, and for the mutual inductance between two parallel conductors. From these expressions, analytical results for the total inductance of loop circuits composed of many straight conductors can simply be assembled by means of linear combination [7]. Since the result is an analytical expression, the computational effort is minimal. Moreover, the application to a particular cross-sectional shape is just a matter of substituting the corresponding GMD value. Analytical solutions are fast and simple to evaluate. They lend themselves well to vectorization. Therefore, they simplify the solution of inverse problems considerably (i.e. finding specific designs of an inductor to achieve a predefined inductance), or they even enable the solution in the first place. But so far, this versatile method suffered from a serious drawback: it seemed to be restricted to uniform distribution of current, i.e. to DC (direct current). In fact, Wang and Tsai explicitly stated that "...as frequency goes up, the GMR and GMD methods no longer hold because of nonuniform distribution of current caused by the skin effect" ([8], p. 1142). Yet in our previous paper we claimed the GMD method to be valid for any frequency, provided the current distribution be properly considered ([5], p. 34).

In section 2 of this paper we first define the GMD for uniform distribution of current. For the most important example, namely, the circular disk, we discuss the substantial difficulties in deriving the analytical formula for the GMD based on its definition, as encountered by Maxwell [1], 
Sommerfeld [4], and Paul [6]. We then extend the GMD method to include skin effect, so that its advantages may be exploited at arbitrary frequencies. We focus on the partial inductance of a single straight conductor [1] - [6]. For simplicity, we will henceforth omit the adjective partial.

In section 3 we derive an exact relation between the GMD and the internal inductance per unit length for infinitely long circularly symmetric conductors. We use it to derive analytical formulae for the GMD in various situations in sections $4-7$. Thereby we cite results of measurements of total inductance reported in the literature and compare them to calculations based on the GMD method.

In section 4 we show how easily the well-known formula for the GMD of a circular disk can be derived with the help of the relation derived in section 3 , in contrast to the efforts required in the approaches adopted by Maxwell, Sommerfeld, and Paul, as mentioned above. Also, the derivation of Maxwell's formula for the GMD of an annulus to itself becomes very easy, as we show in section 5 .

More importantly, we derive an exact analytical expression for the GMD of infinitely long round wires with skin effect in section 6 . We demonstrate numerically that the result obtained from the integral defining the GMD is consistent with this expression. Further, we discuss two approximate formulae based on elementary functions. In section 7 we treat the case of tubular conductors.

In section 8 we introduce an analytical approximation for the GMD of conductors of square cross section with skin effect based on elementary functions. We systematically compare the final results obtained for the total inductance with exact reference values. This permits us to show that the analytical approximation allows to calculate the total inductance of such conductors for frequencies from DC up to $25 \mathrm{GHz}$ to a precision better than $1 \%$. Section 9 presents our conclusions.

\section{Deriving the GMD from its definition}

\subsection{Uniformly distributed current}

The GMD of the conductor cross section to itself is defined via the arithmetic mean of the natural logarithm of the distance $r_{12}$ between any two points $\overrightarrow{r_{1}}$ and $\overrightarrow{r_{2}}$ in the crosssectional surface $S$, of area $|S|$, leading to the fourdimensional integral

$$
\log (G M D)=\frac{1}{|S|^{2}} \iint_{S S} \log \left(r_{12}\right) d \sigma_{1} d \sigma_{2}
$$

When the current is uniformly distributed, as it is at DC, then the GMD is exactly given by integral (1). For circular cross section, this integral is not straightforward to solve. No antiderivatives in terms of elementary or standard special functions are known.

Nevertheless, the result for the GMD of a circular disk to itself is well-known: $\log (G M D)=\log R-1 / 4$. It is being cited in the literature again and again. It was first published by Maxwell ([1], $\S 692,2^{\text {nd }}$ part of example (9)), albeit without a complete derivation. Maxwell started by present- ing the GMD of a point to a circle ([1], §692, example (7)) without giving a proof. We derive it in section 3 . Then he gave the result for the GMD of any figure to an annular disk ([1], §692, example (8)), again without derivation. For the case when the figure is just a single point, the derivation requires the calculation of two two-dimensional integrals and the GMD of a point to a circle. Next, he gave his formula for the GMD of an annulus to itself ([1], §692, $1^{\text {st }}$ part of example (9)). To derive it from the result of his example (8), a four-dimensional integral must be solved using his addition theorem ([1], §691). From the GMD of the annulus he finally derived the GMD of a circular disk to itself by taking the GMD of the annulus to the limit of vanishing inner radius.

It is interesting to note that earlier in his treatise he had calculated the internal inductance of a tubular conductor from the magnetic energy ([1], §685, equation (22)). It seems that he didn't notice the close similarity of this formula with his result for the GMD of an annulus. In any case, he didn't mention a relation between them as we derive it in section 3 .

In view of the importance of the result for the GMD of a circular disk and Maxwell's scarce presentation, we were amazed to find only two rigorous derivations in the literature:

The older one stems from Sommerfeld ([4], §15D). He considered the electrostatic potential produced by an electric charge homogeneously distributed on the surface of an infinitely long circular cylinder. The potential follows from a two-dimensional version of both the Poisson equation and Green's theorem. It is given by the surface integral of the logarithmic potential $\log r$, with $r$ being the radial coordinate. By assigning a particular value to the surface charge density, the integral turns out to be identical to the inner integral of equation (1). For the special case where point 1 coincides with the center of the circular disk, $r_{12}=r$, the integral can easily be done by means of partial integration. The general solution for the potential, on the other hand, can be found as a function of $r$, up to two unknown integration constants, from solving the two-dimensional Poisson equation with the particular value of the surface charge density mentioned above. For $r=0$, i.e. for the special choice of point 1 coinciding with the center, the general solution must reduce to the special one calculated above. Equating the two solutions determines the values of the two hitherto unknown integration constants in the general solution. Inserting them into the general solution and integrating it over the circular disk finally yields the full integral (1), i.e. the logarithm of the GMD of a circular disk. This is a beautiful example of how a difficult integral can be avoided by considering the problem in a different context where it is easier to solve. This derivation involves quite sophisticated reasoning, though.

The second rigorous derivation was published by Paul ([6], pp. 275-278). He first calculated the GMD of a point to a circular disk, for which the integral (1) reduces to two dimensions. Although no antiderivatives are known, the definite integral over the angle is known. (This is the same integral that we use in section 3 to derive the GMD of a 
point to a circle). The result is a logarithmic function, which can be integrated over the radial coordinate to get the GMD between a point and a circular disk. The result, in turn, can be integrated with respect to the point's position over the whole disk to find the GMD of the disk to itself. To do this, one must apply Maxwell's addition theorem ([1], §691).

\subsection{Skin effect}

When skin effect occurs, the current is no longer uniformly distributed over the cross section. To include the skin effect, we must extend definition (1) of the GMD. In an infinitely long conductor in the quasi-static state the current only flows along it, i.e. in z-direction, so that the current density is $\vec{j}=\left(0,0, j_{z}\right)$. For harmonic excitation, there is a complexvalued representation of $j_{z}$. Its surface integral is realvalued and adds up to the total current $I$ :

$$
\int_{S} j_{z} d \sigma=I
$$

Dividing the current density by the total current, we get the density of flow lines $j$ (German: Stromliniendichte),

$$
j=\frac{j_{z}}{I} .
$$

We propose the following modifications of equation (1):

1. The integrand is to be weighted with the product of the complex densities of flow lines $j$ at the two points of integration $\overrightarrow{r_{1}}$ and $\overrightarrow{r_{2}}$, namely, $j\left(\overrightarrow{r_{1}}\right)$ and $j\left(\overrightarrow{r_{2}}\right)$.

(The factor $1 /|S|^{2}$ from equation (1) is then carried by the weighting factors, since for uniform distribution we have $j=1 /|S|$.)

2. For the integral for $\log (G M D)$ to be real-valued, and for the phase argument of the product of the weighting factors $j\left(\overrightarrow{r_{1}}\right)$ and $j\left(\overrightarrow{r_{2}}\right)$ to be identical to their phase difference, one of the factors is to be complex conjugated (denoted by *).

The GMD with skin effect is then defined as

$$
\log (G M D)=\iint_{S} j\left(\overrightarrow{r_{1}}\right) j\left(\overrightarrow{r_{2}}\right)^{*} \log \left(r_{12}\right) d \sigma_{1} d \sigma_{2}
$$

For uniformly distributed current, integral (2) reduces to (1). From a mathematical point of view, equation (2) is the natural way to extend the definition of the GMD to include skin effect for conductors of arbitrary cross section.

We have seen in subsection 2.1 that for circular cross section the integral (1) is not easy to do analytically. For the integral (2) it even appears to be unfeasible. None of the approaches adopted by Maxwell, Sommerfeld, and Paul is then viable anymore. The reason is that the logarithmic functions they obtained as intermediate results and which they integrated over the radial coordinate, are now multiplied by Bessel functions. Not surprisingly, no antiderivatives are known for these integrands, nor in fact is any of the definite integrals.

\section{Deriving the relation}

In this section, we derive the relation between the GMD and the internal inductance per unit length. For infinitely long conductors with circularly symmetric distribution of current, it holds exactly. It simplifies the analytical calculation of the GMD considerably, and, in the case of skin effect, enables it in the first place.

The total inductance of a conductor is the sum of an external and an internal part (irrespective of its cross-sectional shape). Consequently, the internal inductance is the difference between the total and the external one (all measured at the same frequency, of course):

$$
L_{\text {int }}=L-L_{\text {ext }}
$$

We assume the conductor to be infinitely long and the current distribution to be circularly symmetric. The current then produces an external magnetic field that is independent on how the current is radially distributed. This follows from Ampère's law

$$
\vec{\nabla} \times \vec{B}=\mu_{0} \vec{J}
$$

(where $\vec{B}$ is the vector of the magnetic induction, $\mu_{0}$ the magnetic permeability of the vacuum, $\mu_{0}=4 \pi$. $10^{-7} \mathrm{Vs} /(\mathrm{Am})$, and $\vec{\jmath}=\left(0,0, j_{z}\right)$ the current density) by taking the surface integral of equation (4) over a circular disk of radius $r, r>R$, where $R$ is the (outermost) radius of the conductor. With the help of Stokes' theorem we replace the surface integral on the left-hand side by the circulation of $\vec{B}$, which, by virtue of the circular symmetry, is simply $2 \pi r B_{\varphi}$, where $B_{\varphi}$ is the azimuthal (and only) component of $\vec{B}$ in cylindrical coordinates. The surface integral on the right-hand side is $\mu_{0} I$, where $I$ is the total current. Hence the magnetic induction $B_{\varphi}$ at the radial coordinate $r$ outside the conductor is $B_{\varphi}=\mu_{0} I /(2 \pi r)$. Clearly, it does not depend on the radial current density function $j_{z}(r)$. Consequently, neither does the external inductance. Now, by virtue of the skin effect, changing the current distribution implies altering the frequency, and vice versa. Hence neither the external magnetic induction nor the external inductance depends on frequency.

Since the internal inductance vanishes for fully developed skin effect, the external inductance $L_{\text {ext }}$ equals the highfrequency limit $L_{H F}$ of the total inductance (irrespective of the cross-sectional shape),

$$
L_{\text {ext }}=L_{H F},
$$

but only for circularly symmetric shapes does this equation hold irrespectively of the frequency at which $L_{\text {ext }}$ is meas- 
ured (whereas, by definition, $L_{H F}$ is measured at infinite frequency). Only then can we replace $L_{\text {ext }}$ in equation (3) by $L_{H F}$ (where $L_{\text {int }}$ and $L$ are measured at the same arbitrary frequency):

$$
L_{\text {int }}=L-L_{H F} .
$$

Most conductors in the real world are long ones, i.e. their length $l$ is much larger than their cross-sectional dimension (but finite). Their total inductance $L$ is well approximated by Wien's formula,

$$
L \cong \frac{\mu_{0} l}{2 \pi}[\log (2 l)-1-\log (G M D)]
$$

For more accurate formulae for short conductors see [5]. Wien didn't present it in the general form (7), but with the corresponding DC expressions for the GMD of particular cross-sectional shapes already substituted, like e.g. that for a circular disk, see (our) equation (18), or for an annulus ([9], p. 938). For a derivation for the case of a round wire at DC see e.g. Sommerfeld ([4], §15 C, D). To obtain the inductance $L_{H F}$, one must insert the GMD based on the corresponding current distribution at infinite frequency, $G M D_{H F}$ :

$$
L_{H F} \cong \frac{\mu_{0} l}{2 \pi}\left[\log (2 l)-1-\log \left(G M D_{H F}\right)\right]
$$

From the difference (6) we then get the result for the relation between the GMD and the internal inductance per unit length in its general form

$$
\log (G M D)=\log \left(G M D_{H F}\right)-\frac{2 \pi}{\mu_{0}} \frac{L_{i n t}}{l} .
$$

For circularly symmetric conductors of infinite length, this relation holds exactly since, in contrast to equations (7) and (8), it does not explicitly depend on the length anymore. The internal inductance per unit length is just a (welldefined) constant. For other cross-sectional shapes, the relation no longer holds because equation (6) assumes circular symmetry. Consequently, relation (9) is not valid for rectangular cross sections.

To apply the relation to circularly symmetric conductors, we must know $G M D_{H F}$. First, we derive it based on a round wire, and then we show that the same expression also applies to tubes. In a round wire at fully developed skin effect, the current is uniformly concentrated on the very surface of the wire, i.e. along its cross-sectional circumference, which is a circle. Hence the high-frequency limit of the GMD is that of a circle to itself. It can be derived from the GMD of a point to a circle. Let $R$ be the radius and $d$ the distance of the point to the center of the circle. Then the distance $r_{12}$ from that point to a point at the angular position $\varphi$ on the circle is given by the law of cosines as $r_{12}=$ $\sqrt{R^{2}+d^{2}-2 R d \cos \varphi}$. By the one-dimensional version of integral (1) we have

$$
\begin{aligned}
& \log (G M D)= \\
& \frac{1}{2 \pi R} \int_{0}^{2 \pi} \log \sqrt{R^{2}+d^{2}-2 R d \cos \varphi} \operatorname{Rd} d,
\end{aligned}
$$

which can be written as

$$
\begin{aligned}
& \log (G M D)=\log R+ \\
& \frac{1}{4 \pi} \int_{0}^{2 \pi} \log \left[1+\left(\frac{d}{R}\right)^{2}-2 \frac{d}{R} \cos \varphi\right] d \varphi
\end{aligned}
$$

This definite integral is known ([10], integral 4.224, 15):

$$
\int_{0}^{2 \pi} \log \left(1-2 a \cos x+a^{2}\right) d x=\left\{\begin{array}{ll}
0, & a \leq 1 \\
4 \pi \log a, & a \geq 1
\end{array} .\right.
$$

With its help, we find

$$
\log (G M D)=\left\{\begin{array}{ll}
\log R, & d \leq R \\
\log d, & d \geq R
\end{array} .\right.
$$

On the circle $(d=R)$ we have $\log (G M D)=\log R$. Averaging this intermediate result over the whole circle yields the GMD of a circle to itself or, in other words, the required high-frequency limit of the round wire:

or

$$
\log \left(G M D_{H F}\right)=\frac{1}{2 \pi R} \int_{0}^{2 \pi} \log (R) R d \varphi=\log R,
$$

$$
G M D_{H F}=R \text {. }
$$

Now we want to show that the same value for $G M D_{H F}$ also applies to a tube. Let its outer radius be $R$ and its inner one $R_{i}$. Within a full cylinder at fully developed skin effect, the magnetic induction vanishes everywhere, and no current flows. Hence, we can bore out an inner cylinder of any radius $R_{i}<R$ without affecting the fields and the current distribution on the surface. Thus, the current concentrates solely on the outer surface at $r=R$, and hence equation (11) is also valid for tubes. Inserting it into equation (9) we find a simple, yet exact relation between the GMD and the internal inductance per unit length for infinitely long circularly symmetric conductors of (outer) radius $R$ :

$$
\log (G M D)=\log R-\frac{2 \pi}{\mu_{0}} \cdot \frac{L_{\text {int }}}{l} .
$$

This relation allows to bypass the evaluation of the integrals (1) and (2) in the calculation of analytical formulae for the GMD of circularly symmetric conductors. Instead, it relies on the internal inductance per unit length, which is easy to calculate analytically, as we demonstrate in sections $4-7$. 


\section{Round wire at DC}

Our first example of an application of relation (12) to derive an analytical expression for a GMD is an infinitely long round wire of radius $R$ at DC, i.e. with uniformly distributed current. The result is a remarkably simple derivation of the GMD of a circular disk to itself. First, we calculate the magnetic induction $\vec{B}$ within the wire by means of Maxwell's equation (4). As in section 3 we take the surface integral of equation (4) over a circular disk, but this time of radius $r, r \leq R$. The circulation of $\vec{B}$ is still given by $2 \pi r B_{\varphi}$. But the surface integral on the right-hand side of equation (4) is now $\mu_{0} \pi r^{2} j_{z}$, and the current density is $j_{z}=$ $I /\left(\pi R^{2}\right)$. So, we get the magnetic induction $B_{\varphi}$ at the radial coordinate $r$ inside the wire as

$$
B_{\varphi}(r)=\frac{\mu_{0}}{2 \pi} \frac{r}{R^{2}} I, \quad r \leq R
$$

Now the internal inductance can be calculated with the help of the internal magnetic energy $W_{i n t}$ of the wire (for the calculation we can just consider a piece of any length $l$ anywhere within our infinitely long wire, since we are only interested in its internal inductance per unit length, which is well-defined),

$$
W_{\text {int }}=\frac{1}{2 \mu_{0}} \int_{0}^{l} \int_{0}^{2 \pi} \int_{0}^{R} B_{\varphi}(r)^{2} r d r d \varphi d z=\frac{\mu_{0}}{16 \pi} I^{2} l,
$$

and the relation between the magnetic energy and the inductance,

$$
L_{i n t}=\frac{2}{I^{2}} W_{i n t}
$$

The result is the well-known value for the DC internal inductance per unit length of an infinitely long round wire,

$$
\frac{L_{\text {int }}}{l}=\frac{\mu_{0}}{8 \pi}
$$

Substituting it into relation (12) we immediately find the well-known result for the GMD of a circular disk:

$$
\log (G M D)=\log R-\frac{1}{4}
$$

Inserting equation (17) into (7) yields Wiens's formula for the DC inductance of a round wire of finite length $l$ ([9], p. 929),

$$
L \cong \frac{\mu_{0} l}{2 \pi}\left[\log \left(\frac{2 l}{R}\right)-\frac{3}{4}\right] .
$$

Together with the well-known formula for the mutual inductance $M$ between two parallel filaments of length $l$ and distance $d[1]-[6]$,

$$
M=\frac{\mu_{0}}{2 \pi}\left[l \log \left(\sqrt{l^{2}+d^{2}}+l\right)-l \log \mathrm{d}-\sqrt{l^{2}+d^{2}}+d\right]
$$

one can calculate the inductance of a rectangular loop coil composed of straight round wires from the self-inductance $L_{a}$ of a wire of length $a$, the self-inductance $L_{b}$ of a wire of length $b$, the mutual inductance $M_{a b}$ between two filaments of length $a$ and mutual distance $b$, and the mutual inductance $M_{b a}$ between two filaments of length $b$ and mutual distance $a$ by means of the linear combination ([2], p. 318)

$$
L=2\left[L_{a}+L_{b}-\left(M_{a b}+M_{b a}\right)\right] .
$$

This way Wiens's measurements of the inductance of such coils ([9], p. 939) can be compared to calculations, see Table 1 .

Table 1: Measured $\left(L_{\text {exp }}\right)$ and calculated $\left(L_{\text {calc }}\right)$ DC total inductances of rectangular loop coils of length $a$, width $b$, and wire radius $R$, as well as the relative deviation of $L_{\text {calc }}$ from $L_{\text {exp }}$.

\begin{tabular}{cccccc}
\hline $\begin{array}{c}a \\
{[\mathrm{~cm}]}\end{array}$ & $\begin{array}{c}\mathrm{b} \\
{[\mathrm{cm}]}\end{array}$ & $\begin{array}{c}R \\
{[\mathrm{~mm}]}\end{array}$ & $\begin{array}{c}L_{\text {exp }} \\
{[\mathrm{nH}]}\end{array}$ & $\begin{array}{c}L_{\text {calc }} \\
{[\mathrm{nH}]}\end{array}$ & $\begin{array}{c}\text { Deviation } \\
{[\%]}\end{array}$ \\
\hline 25 & 25 & 1.215 & 948 & 960.5 & 1.3 \\
35 & 15 & 1.215 & 918.4 & 928.4 & 1.1 \\
48 & 2 & 1.35 & 566.2 & 578.6 & 2.2 \\
\hline
\end{tabular}

A systematic error analysis of Wien's formula (18) in function of the length-to-radius ratio $l / R$ based on a comparison with exact inductances calculated by numerical integration was presented in [5]. In summary: If $l / R \geq 20$, the error remains below $1.5 \%$ ([5], Fig. 4).

\section{Tubular conductor at DC}

Our next example is an infinitely long tubular conductor at DC, i.e. a conductor of annular cross section with outer radius $R$, inner radius $R_{i}$, and uniform current distribution. In this section, we show how easily the GMD of an annulus can be derived with the help of relation (12). We start again by calculating the magnetic induction within the conductor by means of equation (4). The circulation of $\vec{B}$ remains $2 \pi r B_{\varphi}$, but the surface integral over the current density on the right-hand side of equation (4) now extends over an annulus of inner radius $R_{i}$ and outer radius $r\left(R_{i} \leq r \leq R\right)$ and yields

$$
\mu_{0} \int_{S} j_{z} d \sigma=\mu_{0} \pi\left(r^{2}-R_{i}^{2}\right) j_{z}
$$

while the current density $j_{z}$ is

$$
j_{z}=\frac{I}{\pi\left(R^{2}-R_{i}^{2}\right)} .
$$

The resulting magnetic induction $B_{\varphi}$ as a function of the radial coordinate $r$ within the annular cross section is now 


$$
B_{\varphi}(r)=\frac{\mu_{0}}{2 \pi r}\left(\frac{r^{2}-R_{i}{ }^{2}}{R^{2}-R_{i}^{2}}\right) I, \quad R_{i} \leq r \leq R .
$$

The boundary condition

$$
B_{\varphi}\left(R_{i}\right)=0
$$

is automatically satisfied. It must hold due to equation (4) because no current flows through the hollow part of the tube. With the help of the energy relations (14) and (15) we find the internal inductance from the magnetic induction (19) by means of the integral

$$
L_{i n t}=\frac{\mu_{0}}{2 \pi} \frac{l}{\left(R^{2}-R_{i}^{2}\right)^{2}} \int_{R_{i}}^{R} \frac{1}{r^{2}}\left(r^{2}-R_{i}^{2}\right)^{2} r d r .
$$

(See the note in parenthesis prior to equation (14)). This results in

$$
\frac{L_{i n t}}{l}=\frac{\mu_{0}}{2 \pi}\left[\frac{1}{4} \frac{R^{2}-3 R_{i}{ }^{2}}{R^{2}-R_{i}{ }^{2}}+\frac{R_{i}{ }^{4}}{\left(R^{2}-R_{i}{ }^{2}\right)^{2}} \log \frac{R}{R_{i}}\right] .
$$

When we insert this into relation (12), we already get Maxwell's equation for the GMD of an annulus ([1], §692, $1^{\text {st }}$ part of example (9)), namely

$$
\log \left(\frac{G M D}{R}\right)=\frac{1}{4} \frac{3 R_{i}{ }^{2}-R^{2}}{R^{2}-R_{i}{ }^{2}}-\frac{R_{i}{ }^{4}}{\left(R^{2}-R_{i}{ }^{2}\right)^{2}} \log \frac{R}{R_{i}} .
$$

In the limit $R_{i} \rightarrow 0$ this reduces to the constant $-1 / 4$, thus reproducing the GMD of a circular disk as given by equation (17). Inserting equation (22) into (7) yields Wiens's formula for the DC inductance of a tubular conductor of finite length $l$ ([9], p. 938).

Wien measured the total inductance of three different round tubes of length $0.5 \mathrm{~m}$ and various inner and outer radii directly in a Wheatstone bridge at low frequency ([9], p. 938). In Table 2, his measured results are compared to calculations based on equations (7) and (22).

Table 2: Measured $\left(L_{\text {exp }}\right)$ and calculated $\left(L_{\text {calc }}\right)$ DC total inductances of tubular conductors of outer radius $R$, inner radius $R_{i}$, and length $0.5 \mathrm{~m}$, as well as the relative deviation of $L_{\text {calc }}$ from $L_{\text {exp }}$.

\begin{tabular}{ccccc}
\hline $\begin{array}{c}R \\
{[\mathrm{~mm}]}\end{array}$ & $\begin{array}{c}R_{i} \\
{[\mathrm{~mm}]}\end{array}$ & $\begin{array}{c}L_{\text {exp }} \\
{[\mathrm{nH}]}\end{array}$ & $\begin{array}{c}L_{\text {calc }} \\
{[\mathrm{nH}]}\end{array}$ & $\begin{array}{c}\text { Deviation } \\
{[\%]}\end{array}$ \\
\hline 3.5 & 1.5 & 481.4 & 483.5 & 0.4 \\
5.5 & 4.5 & 426.2 & 426.3 & 0.03 \\
10.0 & 9.5 & 361.7 & 362.2 & 0.1 \\
\hline
\end{tabular}

\section{Round wire with skin effect}

\subsection{Derivation of the GMD}

In the previous two sections, the current distribution was known a-priori, and the magnetic induction could simply be calculated by means of Maxwell's equation (4). For arbitrary frequency, this is no longer the case. This problem either requires the wave equation to be solved in cylindrical coordinates (as can be found in textbooks [4], [11]), or the iterative solution of an integral equation for the current density function [12]. In either case an infinitely long wire is presumed. We follow the first approach and assume that the current and the fields are harmonic in time with angular frequency $\omega$, i.e. their time dependence is $e^{i \omega t}$. Therefore, we follow the usual impedance convention, i.e. the reactance of an inductor with inductance $L$ at angular frequency $\omega$ is given by

$$
\operatorname{Im}(Z)=\omega L
$$

Im refers to the imaginary part, and $Z$ is the impedance of the wire. For infinitely long round wires, the current density divided by its DC value was given by Sommerfeld ([4], equation (21), p. 156, and equation (3d), p. 166). For the current density itself it follows that

$$
j_{z}(r)=\frac{k}{2 \pi R} \frac{\mathrm{J}_{0}(k r)}{\mathrm{J}_{1}(k R)} I, \quad r \leq R,
$$

where we have dropped the wave factor $e^{i(\omega t-k z)}$ for simplicity, and $\mathrm{J}_{0}$ and $\mathrm{J}_{1}$ are the (complex) Bessel functions of first kind and orders 0 and 1, respectively, and $k$ is the wave number within the wire. In the DC limit $k \rightarrow 0$, the fraction with the Bessel functions converges to $2 /(k R)$ ([13], equation 9.1 .7 and $\left.J_{0}(0)=1\right)$, so that the current density assumes its DC value $j_{z}=I /\left(\pi R^{2}\right)$, as it should. In our convention, the wave number is given by

$$
k=\frac{1-i}{\delta}
$$

with $i$ being the imaginary unit and $\delta$ the skin depth,

$$
\delta=\sqrt{\frac{2}{\mu_{0} \sigma \omega}}
$$

where $\sigma$ is the conductivity of the wire. The internal inductance can be calculated analytically ([11], §61). Note that Landau and Lifschitz used cgs units and the time dependence $e^{-i \omega t}$, so that $\operatorname{Im}(Z)=-\omega L$ and $k=(1+i) / \delta$ instead. We transformed their formulae into SI units and adapted them to our convention. From the transformed formula for the total impedance ([11], equation (61.10)), the internal impedance can be calculated in terms of the ratio of the electric field $E_{z}$ to the magnetic induction $B_{\varphi}$, both evaluated at $r=R$ : 


$$
Z_{\text {int }}=\frac{\mu_{0} E_{z}(R)}{2 \pi R B_{\varphi}(R)} l
$$

The result is sometimes expressed in terms of the DCresistance $\mathcal{R}_{0}=l /\left(\pi R^{2} \sigma\right)$ as ([11], equation (61.11))

$$
Z_{\text {int }}=\frac{\mathcal{R}_{0}}{2} k R \frac{\mathrm{J}_{0}(k R)}{\mathrm{J}_{1}(k R)} .
$$

In the literature $Z_{\text {int }}$ is expressed in many ways. The focus of this paper lies on inductance, so we prefer to use a different form that is not expressed in terms of $\mathcal{R}_{0}$. Our formula follows directly from equation (26) and the fields $E_{z}(R)$ and $B_{\varphi}(R)$. Alternatively, it can be derived from equation (27) by applying the definition of the DC resistance $\mathcal{R}_{0}$ above and equations (24) and (25):

$$
Z_{\text {int }}=-\frac{\mu_{0} \omega l}{2 \pi} \frac{i}{k R} \frac{\mathrm{J}_{0}(k R)}{\mathrm{J}_{1}(k R)} .
$$

From $\omega L_{\text {int }}=\operatorname{Im}\left(Z_{\text {int }}\right)$ it immediately follows that

$$
\frac{L_{i n t}}{l}=-\frac{\mu_{0}}{2 \pi} \operatorname{Im}\left[\frac{i}{k R} \frac{\mathrm{J}_{0}(k R)}{\mathrm{J}_{1}(k R)}\right] .
$$

Now we can get an exact analytical result for the GMD with skin effect by inserting equation (29) into relation (12):

$$
\log (G M D)=\log R+\operatorname{Im}\left[\frac{i}{k R} \frac{\mathrm{J}_{0}(k R)}{\mathrm{J}_{1}(k R)}\right] .
$$

It can be shown that in the DC limit $k \rightarrow 0$ the imaginary part of the bracket converges to $-1 / 4$, so that $\log (G M D)$ adopts its DC value given by equation (17), as expected.

The total inductance of a finitely long round wire at arbitrary frequency can be approximated by equation (7) with $\log (G M D)$ given by equation (30), or by its approximate formula (38) based on elementary functions taken from paragraph 6.3.3.

The standard method of calculating the total inductance of round wires with skin effect is to use the well-known approximation for their external inductance ([2], equation (4), or [6], equation (5.18c)),

$$
L_{\text {ext }} \cong \frac{\mu_{0} l}{2 \pi}\left[\log \left(\frac{2 l}{R}\right)-1\right],
$$

to which the internal inductance according to equation (29) is added. Precisely due to relation (12) this procedure yields the same result as the GMD method by equations (7) and (30).

When comparing the two methods, one must keep in mind that equation (31) used in the standard method is only valid for conductors of circularly symmetric cross section, since its derivation is based on the circular symmetry of the external magnetic induction. This is reflected by the explicit occurrence of the radius $R$ in the equation. By contrast, this restriction does not hold for the GMD method because equation (7) is valid for conductors of any cross-sectional shape. In section 8 , for instance, we apply it to conductors of square cross section.

\subsection{Validation of the extended definition of the GMD}

We need to show that the defining integral (2) is consistent with the result (30). The only way to verify this is by evaluating the integral numerically. We take the current density given by equations (23) - (25) for a unit current, and we note that $r_{12}$ is given by

$$
r_{12}=\sqrt{r_{1}^{2}+r_{2}^{2}-2 r_{1} r_{2} \cos \varphi},
$$

where $r_{1}$ and $r_{2}$ are the radii of the two points in the circular disk, and $\varphi$ is the angle between them. Therefore, one of the two angular integrals simply yields the factor $2 \pi$, and the four-dimensional integral (2) reduces to

$$
\begin{aligned}
& \log (G M D)=\frac{|k|^{2}}{2 \pi R^{2}\left|\mathrm{~J}_{1}(k R)\right|^{2}} \\
& \int_{0}^{2 \pi} \int_{0}^{R} \int_{0}^{R} \mathrm{~J}_{0}\left(k r_{1}\right) \mathrm{J}_{0}\left(k r_{2}\right)^{*} \log \left(r_{12}\right) r_{1} r_{2} d r_{1} d r_{2} d \varphi
\end{aligned}
$$

We carried out the numerical integrations with the help of the MATLAB ${ }^{\circledR}$ function integral3. We computed the integral (33) for $\sigma=5.9595 \cdot 10^{7} \Omega^{-1} \mathrm{~m}^{-1}$, corresponding to the resistivity $\rho=1 / \sigma=1.678 \cdot 10^{-8} \Omega \mathrm{m}$ of copper at $20^{\circ} \mathrm{C}([14], T=293 \mathrm{~K})$, a wire radius $R=1 \mathrm{~mm}$, and 1001 logarithmically spaced frequencies between $1 \mathrm{~Hz}$ and $50 \mathrm{MHz}$, and we evaluated the analytical expression (30) for the same parameter values. The maximum deviation between the two sets of values for $\log (G M D)$ occurred at $396 \mathrm{kHz}$ with a value of $4 \cdot 10^{-4} \%$. This small deviation merely reflects the inaccuracy in the numerical evaluation of the Bessel functions and the three-dimensional integral. So, the right-hand sides of equations (30) and (33) seem to be identical. This means that our proposition (2) for applying the concept of GMD to conductors with skin effect is consistent with the result (30).

\subsection{Numerical evaluation of the GMD}

Equation (30) contains Bessel functions of the complex argument $(1-i) x=e^{-i \frac{\pi}{4}} x, x=R / \delta$. Their real and imaginary part can be expressed by Kelvin functions ([13], definition 9.9.1), as is done in most papers on skin effect. But since program languages such as MATLAB ${ }^{\circledR}$ and the freeware Python include subroutines for the numerical evaluation of Bessel functions with complex argument, it is not necessary to transform equation (30) into a form containing Kelvin functions. Instead, equation (30) can be evaluated directly. But not everybody has access to such software. In this section, we present more elementary ways for evaluating the GMD with skin effect. 


\subsubsection{Grover's Table}

The easiest way to take skin effect into account is to consult Grover's Table. Grover has tabulated an unspecified function $T$ of a dimensionless frequency parameter $x$ ([3], Table $52)$, to be used in his high-frequency formula for the total inductance of a round wire ([3], equation (211)). From this equation and our equations (16) and (31), Grover's function $T$ can be identified as the internal inductance with skin effect divided by its DC value. This definition makes the relative internal inductance $T$ dimensionless, independent on the length of the wire, and it restricts its values to the convenient interval $[0,1]$. From the definition of $T$ given above and equations (12) and (16) it follows that

$$
\log \left(\frac{G M D}{R}\right)=-\frac{T}{4}
$$

According to equation (29) the internal inductance can be completely specified in function of one single argument, namely, $k R$. Or, equivalently but more descriptive, in function of the ratio $R / \delta$. According to equations (12) and (34) this also applies to Grover's function $T$. Therefore, he tabulated it in function of a parameter $x \propto R / \delta$. He has also tabulated $x$ as a function of frequency $f$ ([3], Table 53). He calculated this Table with the resistivity $\rho=1.712$. $10^{-8} \Omega \mathrm{m}$, which he claimed to be for copper at $20^{\circ} \mathrm{C}$. But this is erroneous; it is for copper at $25^{\circ} \mathrm{C}([14], T=298 \mathrm{~K})$. The Table of $x(f)$ is for a wire diameter $1 \mathrm{~mm}$. For a diameter of $d \mathrm{~mm}$, just multiply the values by $d$.

With the help of the two Tables $x(f)$ and $T(x)$, and our equation (34), the logarithm of the GMD divided by the wire radius can directly be evaluated for the frequencies $f$ and dimensionless frequency parameters $x$ that are tabulated.

Unfortunately, the Table of $x(f)$ ends at $f=100 \mathrm{kHz}$ with $x=3.3868$, although the Table of $T(x)$ continues up to $x=100$ or $f=87 \mathrm{MHz}$ (for wire diameter $1 \mathrm{~mm}$ ). Grover gave a formula for $x(f)$ ([3], equation (207)), according to which $x \propto R / \delta$, but to us it is not clear what system of units he used. So, in our opinion, the use of his Table of $T(x)$ is not straightforward, at least not for today's readers. From its last line, we know that $x(100 \mathrm{kHz})=3.3868$. With the data given $\left(R=0.5 \mathrm{~mm}, \rho=1.712 \cdot 10^{-8} \Omega \mathrm{m}, f=\right.$ $100 \mathrm{kHz}), \sigma=1 / \rho$, and with the help of equation (25), we can first calculate the ratio $R / \delta$ in SI units, and then the proportionality factor between it and Grover's $x$. We find

$$
x=1.414263 \cdot \frac{R}{\delta} \propto \sqrt{\omega} .
$$

Now $x$ can be calculated for any frequency with the help of equations (25) and (35) in the familiar SI units.

By evaluating equations (30), (34), and (35), we were indeed able to reproduce all the 95 tabulated values of $T(x)$ ([3], Table 52) to an accuracy of $10^{-4}$. This reflects the accuracy of the Table, which is given to 4 decimal places. For intermediate values, interpolation is needed. Grover gave an example of linear interpolation of his Table for the value $x=18.93$ ([3], example 93), but without indicating the resulting accuracy. We interpolated the tabulated values of $T(x)$ for the midpoints between all $x$ values linearly and computed the corresponding exact $T$ values with the help of equations (30), (34), and (35). Figure 1 shows the resulting relative approximation error in function of $x$. The errors in the order of $25 \%$ for the higher frequencies are quite large. But one must bear in mind that for long conductors the internal inductance is much smaller than the external one, so that the error in the internal inductance will not contribute much to the final error in the total inductance. For $l / R=10$ the internal inductance amounts to roughly $10 \%$ of the external inductance, i.e. about a tenth of the error of the internal inductance manifests itself in the total inductance. On the other hand, the fraction of the internal inductance never drops significantly below $2 \%$ up to $l / R=10^{6}$, so for precise calculations the error of the internal inductance always matters.

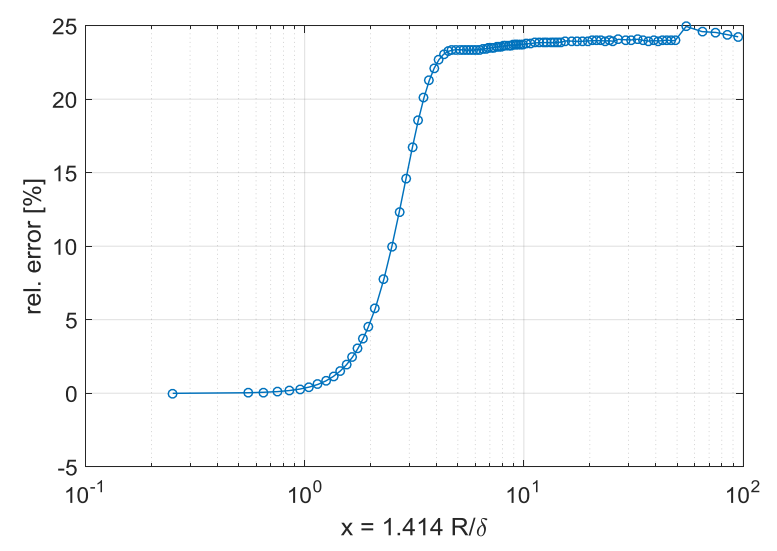

Figure 1: Semi logarithmic plot of the relative error of the linear interpolation of Grover's Table of the relative internal inductance $T(x)$ in function of the dimensionless frequency $x$.

\subsubsection{Wadell's interpolation formula}

Instead of interpolating a Table, it may be more convenient to use an approximate analytical formula. Wadell presented such a formula for $T(x)$ ([15], equation (6.2.1.2)), namely

$$
T(x) \cong \sqrt{\frac{0.00186128 x+0.873011}{0.127964 x^{2}-0.278381 x+1}}, x>0,
$$

where $x$ is given by equation (35). Equation (36) seems to be an optimized formula: When we solved the optimization problem of finding a best fit of the square root of a rational function of total order not exceeding 3 to Grover's Table 52, we got exactly Wadell's formula! (By total order we mean the sum of the nominator and denominator polynomial orders). For large $x$ it approaches zero, as it ought to. But unfortunately, it is not strictly decreasing as it should be. This can be seen in a plot (Fig. 2) or by computing the roots of its derivative. Fig. 2 shows a semi logarithmic plot of function (36), together with the exact curve calculated from 
equation (30) transformed to $T(x)$ by means of equations (34) and (35).

Fig. 3 shows the relative error of equation (36) in function of the dimensionless frequency $x$. Obviously Wadell's formula is not well suited for extrapolating Grover's Table, because for high frequencies its error seems to grow without limit. Of course, it was never intended to be. Nevertheless, because of the shortcomings of equation (36) we tried to find a better formula.

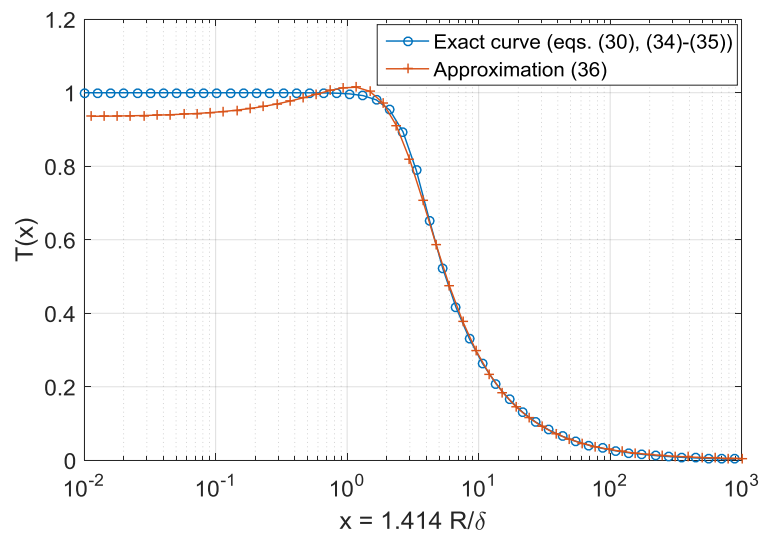

Figure 2: Semi logarithmic plot of the relative internal inductance $T(x)$ in function of the dimensionless frequency $x$.

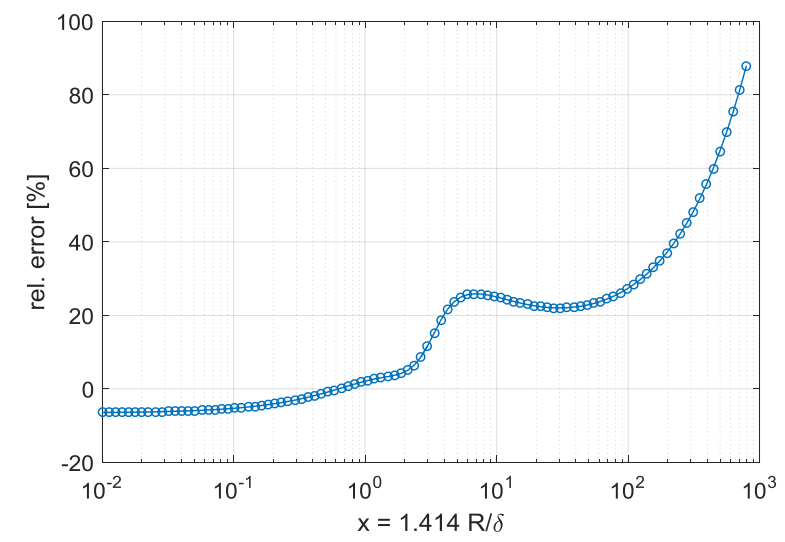

Figure 3: Semi logarithmic plot of the relative error of function (36) for the relative internal inductance in function of the dimensionless frequency $x$.

\subsubsection{Improved interpolation formula}

Instead of $x$ we prefer to use

$$
\zeta=\frac{R}{\delta} \propto \sqrt{\omega}
$$

as the more natural dimensionless frequency parameter, and we want to directly fit $G M D / R$ in function of $\zeta$ instead of its logarithm. We found the following simple formula:

$$
\frac{G M D(\zeta)}{R} \cong\left(1-e^{-\frac{1}{4}}\right)\left[1-\frac{1}{\sqrt[4]{1+\left(p_{0} \zeta\right)^{4}}}\right]+e^{-\frac{1}{4}}
$$

Since it is the logarithm of the GMD that enters the inductance formula, we optimize the parameter $p_{0}$ such that the maximum relative deviation of the logarithm of the function (38) from the exact values given by equation (30) is minimized. This can be done with the help of minimizing functions in MATLAB ${ }^{\circledR}$. Alternatively, for given $p_{0}$, equations (30) and (38) are evaluated for a set of frequencies as defined in subsection 6.2, and the maximum relative deviation between the results of the two equations over all frequencies is calculated. This procedure is repeated for a set of equidistantly placed values of $p_{0}$ in a global interval likely to contain the minimum. The value of $p_{0}$ thus found having the smallest maximum relative deviation represents a first approximation to the solution, provided it does not coincide with one of the endpoints of the global interval. (If it does, the interval must be expanded and the procedure repeated). A new set of equidistant values of $p_{0}$ is defined within a suitable subinterval, and the procedure is repeated, leading to an improved approximation of the solution, etc., until the global minimum is found within the desired accuracy. This results in the value

$$
p_{0}=0.4550
$$

Now function (38) is strictly increasing by design (not decreasing because of the different sign, see equation (34)). Further, it adopts the exact values for $\zeta=0$ and in the limit $\zeta \rightarrow \infty$, namely, $G M D(0) / R=\exp (-1 / 4)=0.7788$ and $\lim _{\zeta \rightarrow \infty} G M D(\zeta) / R=1$. A semi logarithmic plot of the approximate function (38), together with the exact curve, is shown in Fig. 4. The exact curve was calculated by means of equation (30). The approximation (38) is very good: The difference between the two curves is hardly visible.

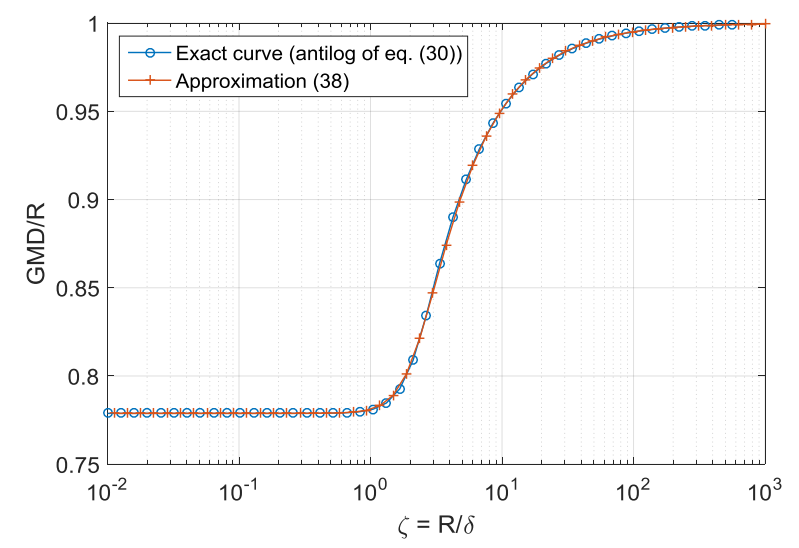

Figure 4: Semi logarithmic plot of the relative GMD in function of the dimensionless frequency $\zeta$.

Fig. 5 shows the relative error of the logarithm of equation (38) in function of the dimensionless frequency $\zeta$, so that it can be compared with Figs. 1 and 3. On comparing it with 
Fig. 3 the improvement of function (38) over (36) becomes apparent.

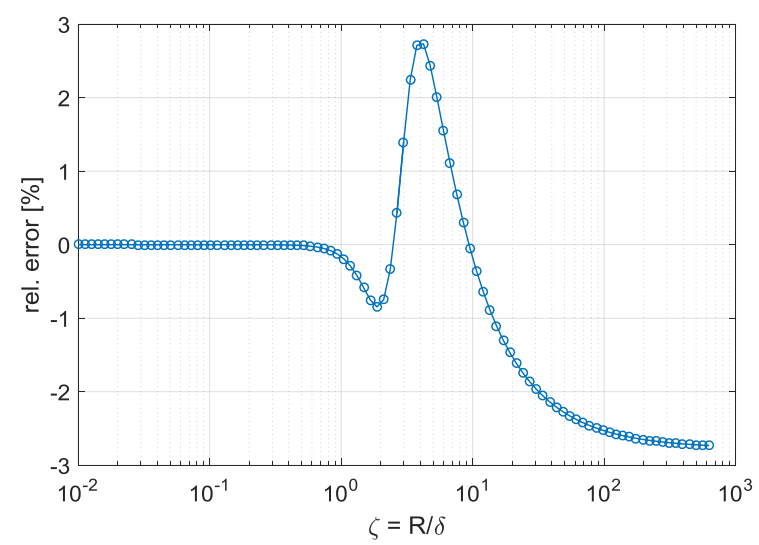

Figure 5: Semi logarithmic plot of the relative error of the logarithm of function (38) for the relative GMD in function of the dimensionless frequency $\zeta$.

\subsection{Comparison to experimental literature data}

Kennelly et al. presented results of precision measurements of the total inductance of a very large rectangular loop coil composed of round copper wires at various frequencies ([16], Table III). Its length was $a=27 \mathrm{~m}$ and the width $b=61.168 \mathrm{~cm}$. The width, i.e. the distance between the cylinder axes of the wires forming the longer sides of the rectangle, resulted from the spacing of $60 \mathrm{~cm}$ between the longer wires and their diameter of $1.168 \mathrm{~cm}$. The measurements were performed between $23.5^{\circ} \mathrm{C}$ at $f=60 \mathrm{~Hz}$ and $27.9^{\circ} \mathrm{C}$ at $f=5000 \mathrm{~Hz}$. Table 3 compares the experimental results with calculations based on the GMD method by equations (7) and (30) for $20^{\circ} \mathrm{C}$, and the equations for the mutual inductance and the linear combination given in section 4 .

Table 3: Measured $\left(L_{\text {exp }}\right)$ and calculated $\left(L_{\text {calc }}\right)$ total inductances of a large rectangular loop coil at various frequencies $f$, and the relative deviation of $L_{\text {calc }}$ from $L_{\text {exp }}$.

\begin{tabular}{ccccc}
\hline $\begin{array}{c}f \\
{[\mathrm{~Hz}]}\end{array}$ & $\begin{array}{c}\zeta \\
{[-]}\end{array}$ & $\begin{array}{c}L_{\text {exp }} \\
{[\mu \mathrm{H}]}\end{array}$ & $\begin{array}{c}L_{\text {calc }} \\
{[\mu \mathrm{H}]}\end{array}$ & $\begin{array}{c}\text { Deviation } \\
{[\%]}\end{array}$ \\
\hline 60 & 0.6939 & 53.912 & 53.808 & -0.2 \\
306 & 1.5670 & 53.767 & 53.658 & -0.2 \\
888 & 2.6693 & 53.143 & 53.045 & -0.2 \\
1600 & 3.5831 & 52.669 & 52.569 & -0.2 \\
2040 & 4.0459 & 52.499 & 52.399 & -0.2 \\
3065 & 4.9592 & 52.215 & 52.156 & -0.1 \\
3950 & 5.6298 & 52.082 & 52.027 & -0.1 \\
5000 & 6.3341 & 51.965 & 51.920 & -0.1 \\
\hline
\end{tabular}

Raising the temperature in the calculations to $25^{\circ} \mathrm{C}$ does not alter the deviations in the last column for the number of decimal places given. For the approximation (36) the deviations happen to be a bit smaller than the tabulated ones based on the exact formula (30), except for $f=306 \mathrm{~Hz}$ and $f=888 \mathrm{~Hz}$ where they are $-0.3 \%$. The reason why the approximation performs so well in these examples is, of course, the large length-to-radius ratio $a / R=4623$ (since $a \gg b$ the ratio $b / R=105$ has less effect). For the approximation (38) all deviations are either the same as in Table 3 or they happen to be smaller.

In their Table, Kennelly et al. didn't give calculated total inductances. Their focus was on the internal inductance. But they gave an example calculation of it ([16], p. 1960). Of course, they used the standard method of separating the total inductance into its internal and external part, and not the GMD method. In their Table, they also used the standard method in the form of (our) equation (3) to find the measured internal inductance by subtracting the calculated external inductance by equation (31) from the measured total inductance. As we justified in subsection 6.1, the standard method yields the same results as the GMD method.

The comparison presented in Table 3 (mainly) tests the calculations for one single value of the length-to-radius ratio, and in a narrow frequency range. In section 8 on conductors of square cross section we test the analytical approximation against numerically calculated exact values. Only when a method is available to calculate exact total inductances is it feasible to do systematic tests for arbitrary parameter combinations in large ranges. Unfortunately, no such method is known for round wires with skin effect.

\section{Tubular conductor with skin effect}

\subsection{Derivation of the GMD}

In this section, we consider an infinitely long tubular conductor of outer radius $R$ and inner radius $R_{i}$ at arbitrary frequency. The singularity at $r=0$ of the Bessel function of the second kind, $Y_{0}(k r)$, lies outside the domain of definition $R_{i} \leq r \leq R$, so that the electric field must now contain both kinds of Bessel functions, in contrast to the case of the round wire ([11], equation (60.2)). Thus, we have for $R_{i} \leq r \leq R$

$$
E_{z}(r)=\left[C \mathrm{~J}_{0}(k r)+D \mathrm{Y}_{0}(k r)\right] e^{i(\omega t-k z)}
$$

with two free constants $C$ and $D$ instead of just one as for the round wire. We use the constant $D$ to impose the boundary condition (20), which must still hold. The magnetic induction $B_{\varphi}$ can be derived from the electric field by means of Faraday's law of induction,

$$
\vec{\nabla} \times \vec{E}=-\frac{\partial \vec{B}}{\partial t}
$$

For cylindrical symmetry with the current flowing only in zdirection, this reduces to

$$
-\frac{\partial E_{z}}{\partial r}=-\frac{\partial B_{\varphi}}{\partial t}=-i \omega B_{\varphi}
$$

Noting that ([13], equation 9.1.28) 
and

$$
\frac{\partial}{\partial r} \mathrm{~J}_{0}(k r)=-k \mathrm{~J}_{1}(k r)
$$

$$
\frac{\partial}{\partial r} \mathrm{Y}_{0}(k r)=-k \mathrm{Y}_{1}(k r)
$$

we find from the electric field (39) and equation (41) the magnetic induction within $R_{i} \leq r \leq R$ as

$$
B_{\varphi}(r)=i \frac{k}{\omega}\left[C \mathrm{~J}_{1}(k r)+D \mathrm{Y}_{1}(k r)\right] e^{i(\omega t-k z)} .
$$

The boundary condition (20) imposes

$$
D=-C \frac{\mathrm{J}_{1}\left(k R_{i}\right)}{\mathrm{Y}_{1}\left(k R_{i}\right)} \text {. }
$$

The ratio of Bessel functions will occur in various formulae in what follows, so we introduce a shortcut for it:

$$
g(k)=\frac{\mathrm{J}_{1}\left(k R_{i}\right)}{\mathrm{Y}_{1}\left(k R_{i}\right)} .
$$

Substituting $g(k)$ in equation (43), inserting the result for $D$ into the fields (39) and (42), and dropping the wave factor since it will be irrelevant in what follows, this yields the following expressions for the fields within $R_{i} \leq r \leq R$ :

$$
\begin{aligned}
& E_{z}(r)=C\left[\mathrm{~J}_{0}(k r)-g(k) \mathrm{Y}_{0}(k r)\right], \\
& B_{\varphi}(r)=i C \frac{k}{\omega}\left[\mathrm{J}_{1}(k r)-g(k) \mathrm{Y}_{1}(k r)\right] .
\end{aligned}
$$

The internal impedance $Z_{\text {int }}$ can again be calculated from equation (26). With the help of the fields (45) and (46), whereby the constant $C$ cancels out, we get

$$
Z_{\text {int }}=-\frac{\mu_{0} \omega l}{2 \pi} \frac{i}{k R} \frac{\mathrm{J}_{0}(k R)-g(k) \mathrm{Y}_{0}(k R)}{\mathrm{J}_{1}(k R)-g(k) \mathrm{Y}_{1}(k R)} .
$$

Lovrić et al. gave a similar result ([17], equation (3)), which can be transformed to our form (47) by means of equations (24), (25), and (44). From $\omega L_{\text {int }}=\operatorname{Im}\left(Z_{\text {int }}\right)$ we find

$$
\frac{L_{i n t}}{l}=-\frac{\mu_{0}}{2 \pi} \operatorname{Im}\left[\frac{i}{k R} \frac{\mathrm{J}_{0}(k R)-g(k) \mathrm{Y}_{0}(k R)}{\mathrm{J}_{1}(k R)-g(k) \mathrm{Y}_{1}(k R)}\right] .
$$

Now we can insert equation (48) into relation (12), and we get an exact analytical result for the GMD of an infinitely long tubular conductor with skin effect:

$$
\begin{aligned}
& \log (G M D)= \\
& \log R+\operatorname{Im}\left[\frac{i}{k R} \frac{\mathrm{J}_{0}(k R)-g(k) \mathrm{Y}_{0}(k R)}{\mathrm{J}_{1}(k R)-g(k) \mathrm{Y}_{1}(k R)}\right],
\end{aligned}
$$

where $g(k)$ is defined by equation (44). For $R_{i}=0$ it follows that $g(k)=0$ ([13], equations 9.1.7 and 9.1.9), and equation (49) reduces to (30) for the round wire.

The total inductance of a finitely long tubular conductor at arbitrary frequency can be approximated with the help of equation (7) with $\log (G M D)$ given by equation (49).

Note that for large arguments $k R$ the numerical evaluation of equations (47) - (49) (and (53)) becomes unstable, also in MATLAB ${ }^{\circledR}$ [17]. The onset of instability with respect to the magnitude of $k R$ depends on the radius ratio $R_{i} / R$. The larger this ratio, the earlier the onset. Lovric et al. presented a special formula for the internal inductance of a tubular conductor claimed to be stable under all conditions [17].

\subsection{Validation of the extended definition of the GMD}

Again, we must verify that our general definition of the GMD is consistent with the result (49), so we must evaluate integral (2) again. To do this, we must know the value of constant $C$. It is determined by the total current,

$$
\int_{0}^{2 \pi} \int_{R_{i}}^{R} j_{Z}(r) r d r d \varphi=I .
$$

The current density is given by Ohm's law as

$$
j_{z}(r)=\sigma E_{z}(r)
$$

Inserting the current density given by equations (45) and (51) into the integral (50) and noting that ([10], integral 5.56, 2 and substitution $x=k r$ )

and

$$
\int \mathrm{J}_{0}(k r) r d r=\frac{r}{k} \mathrm{~J}_{1}(k r)
$$

$$
\int \mathrm{Y}_{0}(k r) r d r=\frac{r}{k} \mathrm{Y}_{1}(k r)
$$

we get the following expression for the constant $C$ :

with

$$
C=\frac{I}{2 \pi} \frac{k}{\sigma p(k)},
$$

$$
\begin{aligned}
p(k) & =R \mathrm{~J}_{1}(k R)-R_{i} \mathrm{~J}_{1}\left(k R_{i}\right) \\
& -g(k)\left[R \mathrm{Y}_{1}(k R)-R_{i} \mathrm{Y}_{1}\left(k R_{i}\right)\right] .
\end{aligned}
$$

Within $R_{i} \leq r \leq R$ the current density is now

$$
j_{z}(r)=\frac{k}{2 \pi p(k)}\left[\mathrm{J}_{0}(k r)-g(k) \mathrm{Y}_{0}(k r)\right] I,
$$

with $g(k)$ according to equation (44). For $R_{i}=0$ we have again $g(k)=0$ in equations (52) and (53), and equation (53) reduces to (23) for the round wire. In the integral (2) one of the angular integrals reduces to the factor $2 \pi$. Thus, we are left with 


$$
\begin{aligned}
& \log (G M D)= \\
& 2 \pi \int_{0}^{2 \pi} \int_{R_{i}}^{R} \int_{R_{i}}^{R} j\left(r_{1}\right) j\left(r_{2}\right)^{*} \log \left(r_{12}\right) r_{1} r_{2} d r_{1} d r_{2} d \varphi,
\end{aligned}
$$

where $j(r)$ is the density of flow lines, $j(r)=j_{z}(r) / I$, with $j_{z}(r)$ from equation (53), and $r_{12}$ is still given by equation (32).

We computed the integral (54) numerically for copper at $20^{\circ} \mathrm{C}$ as in subsection 6.2 , for $R=1 \mathrm{~mm}, R_{i}=0.5 \mathrm{~mm}$, and 1001 logarithmically spaced frequencies between $1 \mathrm{~Hz}$ and $2 \mathrm{MHz}$, and we evaluated the analytical expression (49) for the same parameter values. Beyond $2 \mathrm{MHz}$ the integration with the MATLAB ${ }^{\circledR}$ function integral3 was unsuccessful. This was due to the numerical instability noted at the end of subsection 7.1. Thus, we had to reduce the frequency span compared to the one we had used for the round wire. The maximum deviation between the two sets of values for $\log (G M D)$ occurred at around $1 \mathrm{MHz}$ with a value of $1 \cdot 10^{-4} \%$. This small deviation suggests that the righthand sides of equations (49) and (54) are identical. Thus, our extended definition (2) for the GMD is consistent with the result (49) for infinitely long tubes.

\section{Conductors of square cross section}

\subsection{An alternative approach of finding the GMD}

The application of relation (9) to calculate the GMD of a conductor as described in the previous sections is based on the knowledge of its internal inductance. Unfortunately, no analytical expression for the internal inductance of a conductor of rectangular cross section with skin effect is known. We must relinquish the idea of finding an exact analytical solution in this case and content ourselves with an approximation. To this end we could compute the internal inductance numerically. But this would not help because relation (9) is not valid for rectangular cross sections. Alternatively, we could directly use the defining integral (2). Unfortunately, no analytical expression for the current density is known, so that numerical integration of an analytical integrand as applied in subsections 6.2 and 7.2 is not possible. Numerical integration based on discrete sampling points would be costly (we need sampling points of the current density, which itself requires multi-dimensional integration, before we can do the actual four-dimensional integral) and probably not be very precise.

Fortunately, there seems to be a promising alternative: Restricting ourselves to conductors of square cross section, we calculate several precise reference values of the total inductance numerically. If we do this for a wire that is long enough so that equation (7) is nearly exact, we can use that equation to calculate the corresponding GMD's from these inductances. If we do these calculations for a large enough number of frequencies, we can try to find an analytical function of frequency that fits the GMD's. (Fitting the total inductance directly would require a two-dimensional analytical function, because, unlike the GMD, the inductance also depends on the length of the conductor).

\subsection{Developing the analytical approximation}

The GMD of a conductor of square cross section at arbitrary frequency can be characterized by the cross-sectional side length $s$ and by the frequency. In analogy to the circular case in section 6 , it is to be expected that the GMD can be completely specified by the ratio

$$
\zeta=\frac{S}{\delta} \propto \sqrt{\omega}
$$

For computing the reference values of the total inductance, we used the well-known numerical software FastHenry2, which allows precise computations of the total inductance of conductors of rectangular cross section for arbitrary frequencies [18] and which is available for free download at www.fastfieldsolvers.com. It is based on the cross-sectional subdivision of the conductor into many equal filaments of small but finite rectangular cross-section, all of the same length as the conductor (see also [6]). The conductor is modelled as a parallel connection of magnetically coupled filaments. Thus, the potential differences across each of the filaments are assumed to be identical. The distribution of current within a filament is assumed to be uniform. For this case, exact analytical expressions for the self-inductance of the filaments and for the mutual inductance between spatially separated filaments are known [19]. For the selfinductance of long thin conductors, a numerically more stable formula was developed [20]. The solution is found by solving a system of linear equations involving a huge matrix of impedances. In the literature, this method is used extensively in various forms. It is called by many different, often not very explanatory names. We propose the name finite filaments.

We set the conductance $\sigma$ to $5.9595 \cdot 10^{7} \Omega^{-1} \mathrm{~m}^{-1}$, corresponding to the resistivity $\rho=1 / \sigma=1.678 \cdot 10^{-8} \Omega \mathrm{m}$ of copper at $20^{\circ} \mathrm{C}([14], T=293 \mathrm{~K})$, the length $l$ to $10 \mathrm{~m}$ and the width $s$ to $1 \mathrm{~mm}$. So, we have $l / s=10^{\prime} 000$ and equation (7) is nearly exact. We ran the software for 175 logarithmically distributed frequencies between $50 \mathrm{~Hz}$ and $25 \mathrm{GHz}$ (20 frequencies per decade), corresponding to $\zeta=$ $0.1-2430$. We requested $20 \times 20$ subfilaments. FastHenry 2 then calculated with a total of 3200 filaments. We controlled the accuracy of the results by repeating the calculations at the highest frequency and requesting $30 \times 30$ subfilaments, resulting in a total of 7200 filaments. The results deviated by only 1 unit in the $5^{\text {th }}$ decimal place, so we concluded that the number of filaments was sufficient. With the help of equation (7), very precise reference values for the GMD can then be calculated from the reference inductances. To be able to develop a fitting function that does not depend on a particular cross-sectional size of the wire, we must divide the GMD by some measure of this size. In paragraph 6.3.3 we used $G M D_{H F}$ because this was already predetermined by relation (9) and, luckily, we had the very simple relation $G M D_{H F}=R$. If in rectangular wires the current at infinite frequency were also uniformly distributed along the circumference of the cross section, as it is in round wires, then $G M D_{H F}$ could be calculated analytically. Unfortunately, the current is not uniformly distributed. Rather, it peaks at 
the corners ([6], Fig. 6.14). It seems that, for rectangular cross section, $G M D_{H F}$ cannot be calculated analytically. In contrast, the DC value of the GMD can be calculated analytically. It was given by Maxwell ([1], §692, example (6)). Its calculation by means of integral (1) is tedious but straightforward. For squares, it can be written in the form

$$
G M D(0)=0.447049 \cdot s
$$

Since the procedure to calculate the GMD outlined in subsection 8.1 is not based on relation (9), we are free to choose any base value for the GMD we please. We chose $G M D(0)$. We may expect the resulting fit function $G M D(\zeta)$ to have basically the same characteristics as the corresponding function (38) for round wires (see Fig. 4), but with yet unknown constants $g_{1}$ and $g_{2}$ instead of $1-\exp (-1 / 4)$ and 0.4550 . The resulting fit function has the form

$$
\frac{G M D(\zeta)}{G M D(0)} \cong \mathrm{g}_{1}\left\{1-\frac{1}{\sqrt[4]{1+\left[\mathrm{g}_{2} \cdot \zeta\right]^{4}}}\right\}+1
$$

Clearly, for $\zeta=0$ it must be 1 . Consequently, in contrast to function (38), the last additive constant is 1 instead of $\exp (-1 / 4)$. The values of $g_{1}$ and $g_{2}$ could, in principle, be found by fitting the values of the function (57) for the given 175 frequencies to the reference GMD's divided by $G M D(0)$ given by equation (56). But in the end, it is the logarithm of the GMD that determines the approximated inductance by means of equation (7). Hence, such a fit would lead to a bias in the error curve of the approximated inductance, i.e. the maximum positive deviation from the reference inductances would not be equal to the negative one. As a result, the maximum deviation would be larger than necessary. Yet minimizing the relative deviation of the logarithm of equation (57) from the logarithm of the relative reference GMD's, as we did in subsection 6.3.3 with function (38), is not a good idea because for small frequencies the logarithm of equation (57) is close to zero, and the relative deviation would become very large. It is better to minimize the maximum relative deviation of the approximated total inductances from the reference values calculated with FastHenry2. (In paragraph 6.3.3 we could not do this because no precise values for the total inductance of round wires are available). The approximated total inductance is calculated by multiplying equation (57) by $G M D(0)$ from equation (56) and inserting the result into equation (7), which yields the total inductance. The optimizing problem was solved with the help of a two-dimensional version of the algorithm described in paragraph 6.3 .3 on the 175 frequency samples specified in subsection 8.2. The solution is

$$
g_{1}=0.3212, g_{2}=0.2604 \text {. }
$$

Fig. 6 shows the resulting approximated GMD and its reference in function of the dimensionless frequency.

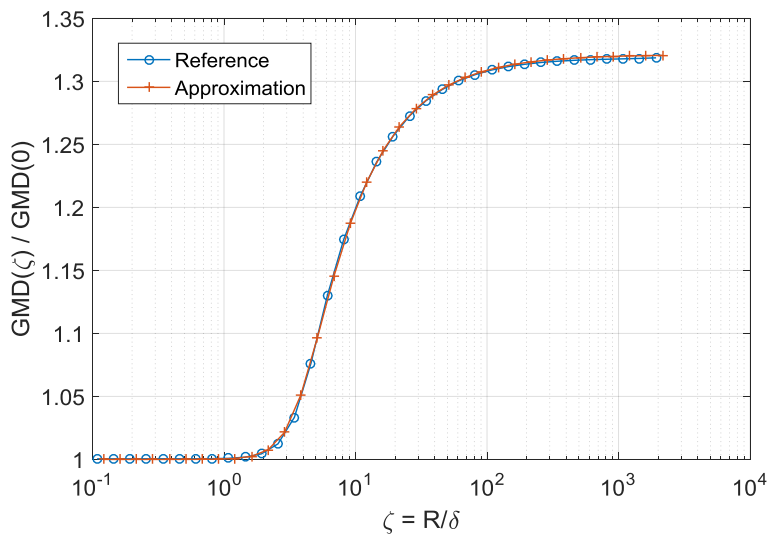

Figure 6: Semi logarithmic plot of the reference and the approximated GMD in function of the dimensionless frequency $\zeta$.

In Fig. 7 we plot the resulting approximated and reference total inductance in function of the dimensionless frequency.

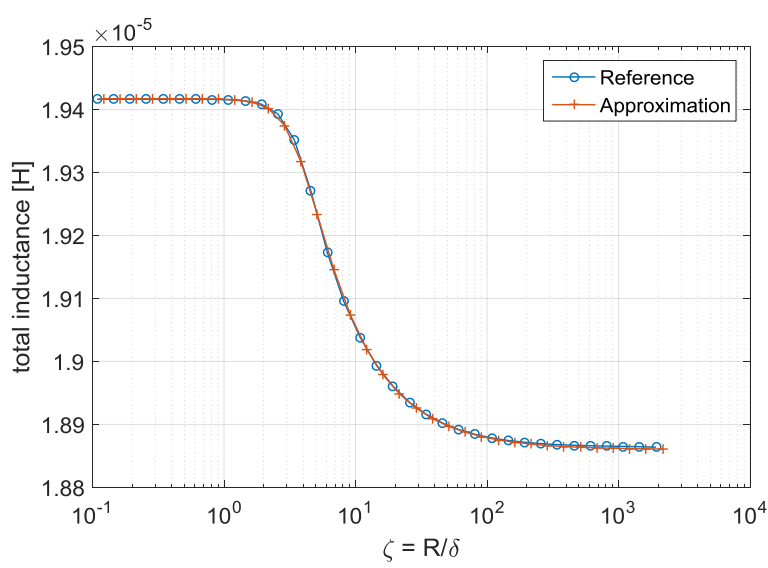

Figure 7: Semi logarithmic plot of the reference and the approximated total inductance in function of the dimensionless frequency $\zeta$.

Figure 8 shows the relative deviation of the calculated total inductances from the reference values in function of the dimensionless frequency. The approximation is excellent: The maximum error is as low as $0.038 \%$ for the 175 data points specified in subsection 8.2, i.e. in the range from $50 \mathrm{~Hz}$ to $25 \mathrm{GHz}$ for a conductor width of $1 \mathrm{~mm}$. The error curve is smooth. It is therefore not to be expected that with a denser set of sampling points a larger error would be found. 


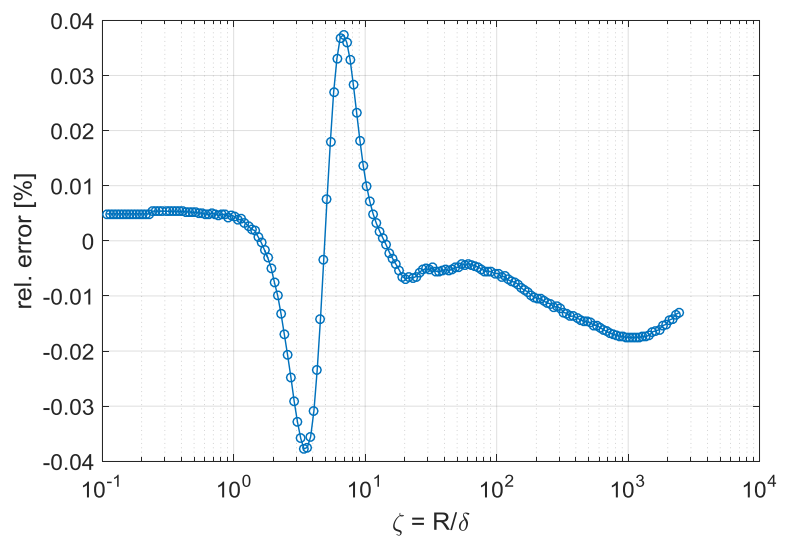

Figure 8: Semi logarithmic plot of the relative error of the approximated total inductance for $l / s=10^{\prime} 000$ in function of the dimensionless frequency $\zeta$.

With the help of this analytical approximation, calculating the total inductance is easy:

1. Calculate the dimensionless frequency $\zeta$ according to equations (25) and (55).

2. Calculate $G M D(0)$ by means of equation (56).

3. Insert $g_{1}, g_{2}$ according to equation (58), and the dimensionless frequency $\zeta$ into function (57) to get the relative GMD.

4. Multiply the relative GMD by $G M D(0)$ from step 2 to get $G M D(\zeta)$.

5. Insert $G M D(\zeta)$ and the length $l$ of the conductor into equation (7) to get the total inductance.

Example (all quantities are in SI-units):

$l=0.3 \mathrm{~m}, s=0.01 \mathrm{~m}, f=4000 \mathrm{~Hz}$ gives $L=2.2329$. $10^{-7} \mathrm{H}$ (exact result: $2.2440 \cdot 10^{-7} \mathrm{H}$, error: $-0.49 \%$ ).

The above steps involve only elementary functions. They can easily be carried out on a programmable pocket calculator.

\subsection{Validation}

In this subsection, we want to determine the precision one can expect in calculating total inductance by means of equations (55) - (57) and (7). Due to its approximate character, the precision of equation (7) will suffer the more the smaller the ratio $l / s$. Hence, we must choose the lowest possible value of this ratio to put the approximation to the test. From our experience with round wires [5] we chose $l=25 \mathrm{~mm}$ and $s=1 \mathrm{~mm}$. We ran FastHenry 2 for the same conditions as in the last subsection, except for $l$. Next, we carried out the five steps listed above for the same values of $\zeta$ to find the approximated inductances.

Finally, we computed the approximation error, i.e. the deviation of the approximated inductances from the reference values computed with FastHenry2. Fig. 9 shows the resulting approximation error for all 175 frequencies.

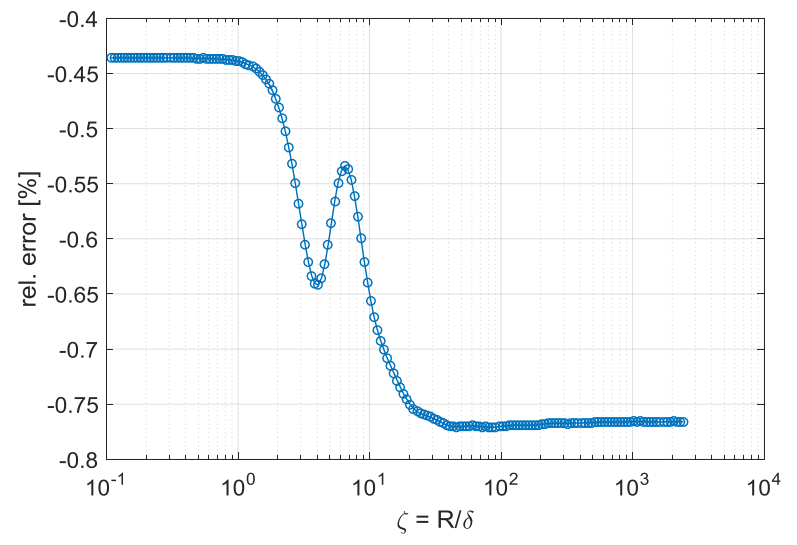

Figure 9: Semi logarithmic plot of the relative error of the approximated total inductance for $l / s=25$ in function of the dimensionless frequency $\zeta$.

The curve has a negative bias. The reason is the approximation error of equation (7) for this low ratio $l / s$. The errors are now roughly ten times larger compared to the case of Fig. 8. The curve is still smooth. The maximum error magnitude now amounts to $0.77 \%$.

From Figs. 8 and 9 we may conclude that our analytical approximation allows to compute the total inductance of conductors of square cross section for $l / s \geq 25$ and for dimensionless frequencies up to 2430 (corresponding to $25 \mathrm{GHz}$ for $s=1 \mathrm{~mm}$ ) to a precision better than $1 \%$.

Owing to its excellent asymptotic behavior, the function (57) can even be used far beyond the frequency range for which it was tested. For instance, for $l=25 \mathrm{~mm}, s=$ $1 \mathrm{~mm}$, and $f=100 \mathrm{GHz}$ (corresponding to $\zeta=4850$ ), the exact inductance according to FastHenry2 is $17.327 \mathrm{nH}$. Our approximation yields $L=17.194 \mathrm{nH}$, which is $-0.77 \%$ off.

The total run time for FastHenry2 to compute all 175 total inductances was about 20 minutes. The MATLAB ${ }^{\circledR}$ implementation of the analytical approximation did it on the same $\mathrm{PC}$ in less than ten milliseconds.

\section{Conclusions}

The GMD method for calculating inductance offers many advantages over other methods: It enables the derivation of general analytical expressions for the total inductance valid for any cross-sectional shape. This shape is considered simply by inserting the corresponding value of the GMD. Expressions for single straight conductors can be extended to encompass loop circuits composed of several conductors by means of linear combination. Analytical expressions simplify the solution of inverse problems. And, above all, they offer unbeatable speed.

But so far, the method seemed to be limited to DC current. We have extended its scope to include skin effect by proposing a modified definition of the GMD. The latter is generally defined by a four-dimensional integral. For circular symmetry and at DC these integrals are demanding to do analytically. With skin effect, they even appear to be unfeasible. It is remarkable that, with the help of our simple rela- 
tion between the GMD and the internal inductance per unit length, their solution becomes so easy.

We have shown that this relation enables short and elementary derivations of Maxwell's analytical expressions for the GMD of circular and annular disks. By contrast, the few derivations found in the literature all require advanced calculus.

With the help of this relation, we have developed exact analytical expressions for the GMD in case of infinitely long round wires and tubular conductors for arbitrary frequencies. We have shown that the numerical results obtained with the extended definition of the GMD are consistent with these expressions. They can directly be used in Wien's formula for the total inductance.

We have found a simple yet precise analytical approximation for the GMD of round wires based on elementary functions.

We have compared the results from our expressions for the GMD of circularly symmetric conductors with measured data from the literature.

Hitherto the calculation of the total inductance of conductors of rectangular cross section with skin effect required costly number crunching on a computer. Our analytical approximation now allows to do calculations for square cross sections within a fraction of a second. They can even be done on a pocket calculator, to a precision better than $1 \%$, irrespective of the size of the conductor.

We have shown that the GMD method can be extended to conductors of circular, annular, and square cross section with skin effect. The formulae presented will enable the derivation of analytical expressions for the total inductance of structures composed of several straight conductors, like shorted two-wire lines and loop coils, taking skin effect into account, provided that adjacent conductors are sufficiently spatially separated to avoid proximity effects. What "sufficiently spatially separated" means in this context is investigated in our later paper [21]. In our comparison of calculated with experimental results in the case of round wires, we have already successfully made use of expressions for the total inductance of rectangular loop coils.

\section{Acknowledgements}

The first author wishes to thank LEGIC Identsystems AG for granting the time to work on this paper.

\section{References}

[1] J. C. Maxwell, A Treatise on Electricity and Magnetism, vol. 2, Clarendon Press, Oxford, 1873. Downloadable at https://archive.org.

[2] E. B. Rosa, The Self and Mutual Inductances of Linear Conductors, Bulletin of the Bureau of Standards, vol. 4, no. 2, Washington, 1907.

[3] F. W. Grover, Inductance Calculations, Dover Publications, New York, 2009, first published by D. Van Nostrand Co., New York, 1946.

[4] A. Sommerfeld, Elektrodynamik, Verlag Harry Deutsch, Frankfurt am Main, 1988, $4^{\text {th }}$ ed. 2001.
[5] H. A. Aebischer and B. Aebischer, Improved Formulae for the Inductance of Straight Wires, Advanced Electromagnetics, vol. 3, no. 1, 31-43, 2014, DOI: http://dx.doi.org/10.7716/aem.v3i1.254

[6] C. R. Paul, Inductance, John Wiley \& Sons, Hoboken NJ, 2010.

[7] H. M. Greenhouse, Design of Planar Rectangular Microelectronic Inductors, IEEE Trans. on Parts, Hybrids, and Packaging, vol. 10, no. 2, 101-109, 1974.

[8] Y. J. Wang and Y. L. Tsai, Calculation of the Frequency-Dependent Impedance of Rail Tracks Using a FourParameter Equivalent Tubular Conductor Model, IEEE Trans. on Power Delivery, vol. 19, no. 3, 1142-1147, 2004.

[9] M. Wien, Ueber die Berechnung und Messung kleiner Selbstpotentiale, Wiedemanns Annalen 53 (Annalen der Physik 289), 928-947, 1894.

[10] I. S. Gradshteyn and I. M. Ryzhik, Table of Integrals, Series, and Products, Academic Press, Amsterdam, $7^{\text {th }}$ ed. 2007.

[11] L. D. Landau and E. M. Lifschitz, Elektrodynamik der Kontinua, Akademie-Verlag Berlin, Berlin, $5^{\text {th }}$ ed. 1985, first published by edition NAUKA, Moscow, 1982.

[12] G.S. Smith, A Simple Derivation for the Skin Effect in a Round Wire, European Journal of Physics, vol. 35, $1-13,2014$.

[13] M. Abramowitz and I. A. Stegun, Handbook of Mathematical Functions, National Bureau of Standards, Washington DC, 1964.

[14] W. M. Haynes, Th. J. Bruno, and D. R. Lide, CRC Handbook of Chemistry and Physics, $95^{\text {th }}$ ed., Internet Version 2015, p. 12-41, 2015.

[15] B. C. Wadell, Transmission Line Design Handbook, Artech House, Boston, 1991.

[16] A. E. Kennelly, F. A. Laws and P. H. Pierce, Experimental Researches on Skin Effect in Conductors, Transactions of the American Institute of Electrical Engineers, vol. XXXIV, no. 2, 1953-2021, 1915.

[17] D. Lovrić, V. Boras, and S. Vujević, Accuracy of Approximate Formulas for Internal Impedance of Tubular Cylindrical Conductors for Large Parameters, Progress in Electromagnetics Research M, vol. 16, 171-184, 2011.

[18] M. Kamon, M. J. Tsuk, and J. K. White, FASTHENRY: A Multipole-Accelerated 3-D Inductance Extraction Program, IEEE Trans. on Microwave Theory and Techniques, vol. 42, no. 9, 1750-1758, 1994.

[19] C. Hoer and C. Love, Exact Inductance Equations for Rectangular Conductors With Applications to More Complicated Geometries, Journal of Research of the 
National Bureau of Standards, vol. 69C, no. 2, 127137, 1965.

[20] A. E. Ruehli, Inductance Calculations in a Complex Integrated Circuit Environment, IBM Journal of Research and Development, 470-481, September 1972.

[21] H. A. Aebischer and H. Friedli, Analytical Approximation for the Inductance of Circularly Cylindrical TwoWire Transmission Lines with Proximity Effect, submitted for publication in Advanced Electromagnetics, May 2017. 